\title{
Epiblepharon with inverted eyelashes in Japanese children. I. Incidence and symptoms
}

\author{
SACHIKO NODA, SEIJI HAYASAKA, AND TOMOICHI SETOGAWA \\ From the Department of Ophthalmology, Shimane Medical University, Izumo, Japan
}

SUMMARY Epiblepharon commonly occurs in Japanese infants and tends to disappear spontaneously with age. We examined 4449 Japanese children aged 3 months to 18 years for epiblepharon associated with inverted eyelashes touching the cornea. The condition was evident in 441 cases. We found that the incidence of epiblepharon decreased with age, but about $2 \%$ of high school students still had the condition. No sexual predilection was found. Lower eyelids were commonly involved bilaterally. Most cases of epiblepharon produced no or mild symptoms.

Epiblepharon is characterised by a fold of skin that stretches horizontally across the upper or lower eyelid, usually associated with inversion of eyelashes. ${ }^{1}$ It is reported to be common in infants of Oriental races. ${ }^{\prime}$ To our knowledge no report details the incidence of this condition in recent Japanese literature. We therefore examined the incidence and symptoms of epiblepharon in Japanese children.

\section{Subjects and methods}

A total of 4449 Japanese children in Shimane Prefecture were examined. Infants were seen on routine 3-month or 6-month screening tests at health centres. Children aged 1 to 6,7 to 12 , and 13 to 18 years were examined on routine ophthalmic screening at nurseries, elementary schools, and high schools, respectively.

Epiblepharon was diagnosed as an extra fold of eyelid skin associated with inverted eyelashes touching the corneal surface. The condition was observed with a binocular loup. Corneal touch was confirmed by topical staining with fluorescein and was inspected in forward, upward, downward, nasal, and temporal eye positions. Epicanthus-like conditions were included in the diagnosis when inverted eyelashes produced corneal touch, because the condition was too difficult to differentiate from epiblepharon. Children who had undergone surgery for epiblepharon were also included, even though the condition had been resolved.

Correspondence to Seiji Hayasaka, MD, Department of Ophthalmology, Shimane Medical University, Izumo, Shimane 693, Japan.
Excluded were cases of true entropion of the eyelid, trichiasis, and distichiasis. To differentiate true entropion, the fold or fold-like skin was pulled down in the lower eyelid or up in the upper eyelid. In cases of epiblepharon the eyelashes were turned out and the normal location of the eyelid margin became visible. In true entropion the entire eyelid margin was pulled outward. Also excluded were cases of corneal abrasion produced by eyelashes associated with microcornea and buphthalmos.

\section{Results}

Of 4449 children examined 441 had epiblepharon with inverted eyelashes touching the cornea (Table 1). Four had already undergone surgery. Of 308 infants $142(46 \%)$ were diagnosed with epiblepharon. The incidence of the condition decreased with age: $24 \%$ at age 1 year, $20 \%$ at age 2 years, $17 \%$ at ages $3-4,7 \%$ at ages $5-6$, and $2 \%$ at ages $13-18$. No sexual predilection was found. Table 2 shows the eyelid involvement of all cases of epiblepharon. Lower eyelids were commonly involved bilaterally.

Table 3 indicates clinical symptoms in the 441 cases of epiblepharon. Most children had no complaints (78\%) despite corneal touch. The most frequent complaints were tearing or discharge, photophobia, and discomfort or foreign body sensation.

\section{Discussion}

It is difficult to confirm the presence of epiblepharon in Japanese children by inspection of the eyelid skin only. We therefore considered an extra fold of eyelid 
Table 1 Age and sex of children with epiblepharon

\begin{tabular}{|c|c|c|c|c|}
\hline Age (yr) & Sex & $\begin{array}{l}\text { No. of children } \\
\text { examined }\end{array}$ & $\begin{array}{l}\text { No. of cases of } \\
\text { epiblepharon }\end{array}$ & Percent \\
\hline \multirow[t]{2}{*}{0} & $M$ & 163 & 73 & $44 \cdot 8$ \\
\hline & $\mathrm{F}$ & 145 & 69 & $47 \cdot 6$ \\
\hline \multirow[t]{2}{*}{1} & $\mathbf{M}$ & 152 & 40 & $26 \cdot 3$ \\
\hline & $F$ & 163 & 36 & $22 \cdot 1$ \\
\hline \multirow[t]{2}{*}{2} & $\mathbf{M}$ & 102 & 21 & $20 \cdot 6$ \\
\hline & $\mathrm{F}$ & 115 & 22 & $19 \cdot 1$ \\
\hline \multirow[t]{2}{*}{$3-4$} & $\mathbf{M}$ & 287 & 44 & $15 \cdot 3$ \\
\hline & $F$ & 251 & 46 & $18 \cdot 3$ \\
\hline \multirow[t]{2}{*}{$5-6$} & $\mathbf{M}$ & 127 & 9 & $7 \cdot 1$ \\
\hline & $F$ & 101 & 7 & $6 \cdot 9$ \\
\hline \multirow[t]{2}{*}{$7-9$} & $\mathbf{M}$ & 353 & 15 & $4 \cdot 2$ \\
\hline & $\mathrm{F}$ & 333 & 14 & $4 \cdot 2$ \\
\hline \multirow[t]{2}{*}{$10-12$} & $\mathbf{M}$ & 251 & 4 & $1 \cdot 6$ \\
\hline & $\mathrm{F}$ & 250 & 7 & $2 \cdot 8$ \\
\hline \multirow[t]{2}{*}{$13-15$} & $\mathbf{M}$ & 123 & 3 & $2 \cdot 4$ \\
\hline & $F$ & 129 & 3 & $2 \cdot 3$ \\
\hline \multirow[t]{2}{*}{$16-18$} & $\mathbf{M}$ & 683 & 13 & 1.9 \\
\hline & $\mathbf{F}$ & 721 & 15 & $2 \cdot 1$ \\
\hline \multirow[t]{2}{*}{$0-18$} & $\mathbf{M}$ & 2241 & 222 & 9.9 \\
\hline & $\mathrm{F}$ & 2208 & 219 & 9.9 \\
\hline Total & & 4449 & 441 & 9.9 \\
\hline
\end{tabular}

Table 2 Eyelid involvement in 441 cases of epiblepharon

\begin{tabular}{lc}
\hline Eyelid involved & No. of cases $(\%)$ \\
\hline Unilateral or bilateral: & \\
Unilateral & $92(21)$ \\
Bilateral & $349(79)$ \\
Upper or lower: & \\
Upper only & $31(7)$ \\
Lower only & $357(81)$ \\
Upper and lower & $53(12)$ \\
Total & $441(100)$ \\
\hline
\end{tabular}

Table 3 Symptoms in 441 cases of epiblepharon*

\begin{tabular}{ll}
\hline Symptoms & No. of cases $(\%)$ \\
\hline No & $344(78)$ \\
Yes: & $97(22)$ \\
Tearing or discharge & 91 \\
Photophobia & 84 \\
Discomfort or foreign body sensation & 70 \\
Ocular pain & 4 \\
Blurred vision & 4 \\
\hline
\end{tabular}

*Some cases had multiple complaints.

skin plus inverted eyelashes touching the cornea as diagnostic criteria.

During the study we found one case of true entropion, four of trichiasis, one of microphthalmos, and one of buphthalmos in which inverted eyelashes rubbed against the globe. In some children it was very difficult to differentiate epicanthus from epiblepharon. Epiblepharon, especially superiorly, may be considered an exaggeration of epicanthus tarsalis. ${ }^{2}$

Our study demonstrated the following findings: (1) epiblepharon commonly occurs in Japanese infants; (2) the condition tends to disappear spontaneously with age; (3) no sexual predilection is evident; (4) lower eyelid involvement is commonly bilateral; and (5) most cases produce no or mild symptoms.

The terminology used to describe entropion, epiblepharon, and epicanthus has been somewhat controversial in Japan. In 1940 Hirose $^{3}$ suggested that the inverted eyelashes found in many Japanese infants was a condition distinct from true entropion, and he called it shomo-naihan (Japanese) or entropium ciliare (Latin). Since then entropium ciliare has been widely accepted by ophthalmologists in $\mathrm{Japan}^{45}$ to indicate cases of epiblepharon and some cases of epicanthus. The cases of epiblepharon we observed in the present study represented those of entropium ciliare.

The incidence of epiblepharon in the Japanese population has not been reported recently to our knowledge. Oshima and Nishikawa ${ }^{5}$ cited Hashimoto's report from 1926, which indicated that $52.5 \%$ of Japanese infants had inverted eyelashes. Similar results ( $46 \%$ of the infants) were obtained in the present study.

As shown in our study, most cases of epiblepharon produced no or mild complaints, even though the inverted eyelashes touched the cornea. The corneal touch, however, did not always cause superficial keratitis. This finding may be explained by the fineness of the lashes and the tolerance of the cornea, as described by Duke-Elder.' Cases of epiblepharon that produce no clinical symptom require no treatment. Patients complaining of ocular pain and blurred vision should be treated appropriately.

\section{References}

1 Duke-Elder S. Epiblepharon. In: Duke-Elder S, ed. System of ophthalmology. London: Kimpton, 1964; 3: 857-9.

2 Johnson CC. Epicanthus and epiblepharon. Arch Ophthalmol 1978; 96: 1030-3

3 Hirose K. Entropium cutaneum (entropium ciliae cutaneum) and its surgical correction. Nippon Ganka Gakkai Zasshi 1940; 44: 1802-13.

4 Yamamoto M. Shomo-naihan or entropium ciliare. Ganka 1980; 22: 915-9.

5 Oshima T, Nishikawa T. Shomo-naihan or entropium ciliare. Ganka 1986; 28: 813-20.

Accepted for publication 28 January 1988. 